\title{
Synthesis of quercetin-functionalized silver nanoparticles by rapid one-pot approach
}

\author{
Sureshbabu Ram Kumar Pandian ${ }^{1 *}$, Selvaraj KUnJiaPPan ${ }^{1}$, \\ VIGNESHWARAN RAVISHANKAR ${ }^{2}$, VELMURUGAN SUNDARAPANDIAN ${ }^{1}$ \\ ${ }^{1}$ Department of Biotechnology, Kalasalingam Academy of Research and Education, Krishnankoil, Tamil Nadu, India \\ ${ }^{2}$ Department of Biotechnology, Mepco Schlenk Engineering College, Sivakasi, Tamil Nadu, India
}

\begin{abstract}
Nanotechnology plays a pivotal role in all areas of science, and the synthesis and surface modification of nanoparticles are crucial in this field. Metal nanoparticles synthesized using plant extracts or phytocompounds are highly significant in the development of various therapeutics and diagnostics. In the present study, we report the one-pot and rapid synthesis of silver nanoparticles (AgNPs) by quercetin, a flavonoid found in various edible plants. The nanoparticles were then characterized using UV-VIS spectrophotometry, Fourier-transform infrared spectroscopy (FT-IR), scanning electron microscopy (SEM), and transmission electron microscopy (TEM). The role of quercetin in the synthesis of silver nanoparticles was demonstrated. The nanomaterial was $20-30 \mathrm{~nm}$ in size and spherical in shape. The silver nanoparticles were also confirmed to show antioxidant activity at $80 \mu \mathrm{g} / \mathrm{ml}$ concentration in vitro by DPPH, FRAP, and power reduction methods.
\end{abstract}

Key words: quercetin, bioflavonoid, one-pot synthesis, AgNPs, antioxidant

\section{Introduction}

Metal nanoparticles are considered to be efficient in various biomedical applications as compared to other nanomaterials (Azharuddin et al., 2019). Biological synthesis of nanoparticles has been considered as an ecofriendly and natural approach (Ingale and Chaudhari, 2013), wherein herbal/medicinal plants have been used widely. Given its rapid, environmental-friendly, economi$\mathrm{cal}$, and precise one-step approach, the fabrication of metal nanoparticles using plant extracts has gained significant attention (Ahmed et al., 2016). Various phytocompounds such as polysaccharides, alkaloids, tannins, phenolics, saponins, and terpenoids are used in the salt reduction method for nanoparticle synthesis (Ahmed et al., 2016). Plant metabolites are excellent reducing agents and thus exhibit the potential to convert the salt into nanoparticles (Mittal et al., 2013). Furthermore, the phytosynthesis and purification of nanomaterials are simpler than other methods (microbial- and/or chemical- mediated) (Mittal et al., 2013). For instance, the leaf extract of Sesbania aegyptiaca has been used to synthesize PEGylated silver nanoparticles, and its immunomodulatory and antioxidant properties were demonstrated in vitro (Pandian et al., 2018). Moreover, the synthesis of plant-based nanomaterials also offers good biocompatibility and fabrication of applicable nanomaterials (Borase et al., 2014).

Interestingly, various parts of plants have been used for nanoparticle synthesis (Borase et al., 2014), including leaf extracts of Combretum erythrophyllum (Jemilugba et al., 2019), stem extracts of Phyllanthus pinnatus (Balachandar et al., 2019), root extract of Berberis vulgaris (Behravan et al., 2019), and fruit extracts of Phyllanthus emblica (Masum et al., 2019). Quercetin is a widely distributed natural bioflavonoid in various medicinal plants (Baková and Kolesárová, 2020). It possesses antioxidant, anti-inflammatory (Lesjak et al., 2018), anticancer, and neuroprotective (Dajas, 2012) properties. It

\footnotetext{
* Corresponding author: Department of Biotechnology, School of Bio and Chemical Engineering, Kalasalingam Academy of Research and Education, Krishnankoil-626126, Tamil Nadu, India; e-mail: srkpandian@gmail.com
} 
has also been proven as a potential reducing agent in the synthesis of therapeutic nanomaterials (Lakshmi and Kim, 2019).

The stability of synthesized nanomaterials is influenced by the concentration of salt (core material) and reducing agents (Virkutyte and Varma, 2011). In this sense, various parameters have been used to optimize nanomaterial synthesis, including the reducing material $\left(\mathrm{Ag}^{+}\right.$to $\left.\mathrm{Ag}^{0}\right)$ (Pandian et al., 2010), salt concentration, and physical parameters $(\mathrm{pH}$ and temperature); these factors together influence the size and shape of nanoparticles (Nikaeen et al., 2020). For instance, many studies that adopted various $\mathrm{pH}$ values concluded that $\mathrm{pH}$ 7 is optimal for the synthesis of AgNPs (Bawaskar et al., 2010; Krishnaraj et al., 2012; Rajan et al., 2015). Eventually, temperature between 25 and $40^{\circ} \mathrm{C}$ was considered suitable for the synthesis of AgNPs (Bawaskar et al., 2010; Saxena et al., 2016).

Given the nontoxic characteristic of biogenic metal nanoparticles (Seralathan et al., 2014), it was widely used in various biomedical applications (Singh et al., 2017). AgNPs are advantageous because of their unique properties such as optical (Evanoff Jr and Chumanov, 2005), electronic (Chen et al., 2009), biosensing (Lin et al., 2009), antibacterial (Ren et al., 2019), antioxidant (Taha et al., 2019), and anticancer (Anandan et al., 2019) properties. In particular, colloidal silver has been widely chosen for therapeutic applications as compared to other forms of nanosilver (Wei et al., 2015).

The synthesized nanoparticles are characterized using various instrumentation methods, and their biomedical efficacy is established. In line with this, $\mathrm{MgO}$ nanoparticles were synthesized using aqueous leaf extracts $P$ sidium guvajava and Aloe vera, and characterized by UVVIS spectroscopy, Fourier-transform infrared spectroscopy (FT-IR), X-ray powder diffraction (XRD), field emission scanning electron microscopy (FESEM), energy-dispersive X-ray spectroscopy (EDAX), and X-ray photoelectron spectroscopy (XPS) (Umaralikhan and Jaffar, 2018). The antibacterial activity of the $\mathrm{MgO}$ nanoparticles was also studied. The results revealed that $5 \mathrm{mg} / \mathrm{ml}$ of $\mathrm{MgO}$ nanoparticles were effective in inhibiting $88 \%$ bacterial growth (Umaralikhan and Jaffar, 2018). Silver and gold nanoparticles were fabricated using the extract of Phoenix dactylifera, and the physicochemical properties were further characterized (Banu et al., 2018). Their anticancer activity on the MCF-7 breast cancer cell line was tested, which revealed a dose-dependent cytotoxicity by enhancing the expression of the antiapoptotic protein Bcl-2 and the proapoptotic protein p53 (Banu et al., 2018). The antioxidant properties of AgNPs synthesized with the extract of Lavandula stoechas were determined by (Mahmoudi et al., 2020). The synthesized AgNPs were then proved to exhibit antioxidant $(125 \mu \mathrm{g} / \mathrm{ml})$, antibacterial $(125 \mu \mathrm{g} / \mathrm{ml})$, and cytotoxic $(500 \mu \mathrm{g} / \mathrm{ml})$ properties in vitro (Mahmoudi et al., 2020).

In the present study, AgNPs were synthesized by the rapid one-pot method by using quercetin, a flavonoid found in many plants and red wine. The synthesized nanoparticles were then characterized by UV-VIS spectrophotometry, scanning electron microscopy (SEM), and transmission electron microscopy (TEM). The in vitro antioxidant potential of quercetin-functionalized NPs was also demonstrated.

\section{Materials and methods}

\section{Materials}

Quercetin (Q4951-10G) was purchased from SigmaMerck, Bengaluru, India. All other chemicals were obtained from Hi-Media Laboratories Pvt. Ltd., Mumbai, India.

\section{One-pot synthesis of AgNPS}

The stock solution of quercetin $(2 \mathrm{mM})$ was prepared using $1 \mathrm{mM}$ sodium hydroxide solution at $\mathrm{pH} 8$ and stored at room temperature. Next, $1 \mathrm{mM}$ of $10 \mathrm{ml}$ silver nitrate was prepared, to which $0.1 \mathrm{ml}$ of quercetin solution was added in a drop-wise manner under constant stirring at room temperature. This facilitated the bioreduction of silver ions $\left(\mathrm{Ag}^{+}\right)$to neutral ions $\left(\mathrm{Ag}^{0}\right)$ in the solution, which was evidenced by the appearance of brown color. The resultant colloidal AgNPs were filtered through a 0.22 -micron syringe filter and stored in refrigerator $\left(2-8^{\circ} \mathrm{C}\right)$ until further analysis. The experiment was repeated three times, and the reliability of this method was confirmed.

\section{UV-VIS spectrophotometry}

The biosynthesis and reduction of silver ions to AgNPs were initially characterized on the basis of their absorbance spectra by a UV-VIS spectrophotometer (JASCOV-650 spectrophotometer) between the range of 300-700 nm. 


\section{SEM analysis}

The size and morphology of the synthesized AgNPs were determined using a scanning electron microscope (Carl Zeiss EVO 18) at an accelerating voltage of $20 \mathrm{kV}$. The nanoparticle solution was diluted 5-fold with deionized water. Subsequently, a few drops of dispersed AgNPs were placed on an aluminum stub, which was airdried at $60^{\circ} \mathrm{C}$ for $5 \mathrm{~min}$. The sample was then scanned, and photomicrographs were captured.

\section{TEM analysis}

The biosynthesized AgNPs were morphologically characterized by a transmission electron microscope (JEOL model 2100, Japan). The microscope was operated at an optimum accelerating voltage of $200 \mathrm{kV}$. A few drops of re-dispersed AgNPs were placed on a carboncoated copper TEM grids and allowed for $10 \mathrm{~min}$ to settle down, followed by observation under the microscope.

\section{FT-IR}

The integrity of functional groups $(-\mathrm{COOH}, \mathrm{OH}$, $-\mathrm{CH}_{3}$, and $-\mathrm{CO}$ of quercetin) in quercetin and the biosynthesized AgNPs was analyzed using a Fourier-transform infrared spectrophotometer (Shimadzu IR Tracer100). One milligram of lyophilized quercetin and AgNPs was individually mixed with FT-IR grade $\mathrm{KBr}$ and subjected to hydraulic press to prepare a round disc. Subsequently, the discs were used in the spectrometer, in which the spectral ranges were set between 400 and $4000 \mathrm{~cm}^{-1}$.

\section{Antioxidant activities of AgNPS}

\section{DPPH radical scavenging assay}

The antioxidant potential of the synthesized AgNPs was estimated using the DPPH radical scavenging assay (Arora and Chandra, 2011). Briefly, $0.5 \mathrm{ml}$ of various concentrations of AgNPs $(10,20,40,80$, and $160 \mu \mathrm{g} / \mathrm{ml})$ was mixed with $1 \mathrm{ml}$ of $0.1 \mathrm{mM}$ DPPH (2,2,1-diphenyl-1picrylhydrazyl) solution in ethanol, followed by vigorous shaking and incubation at $37^{\circ} \mathrm{C}$ for $30 \mathrm{~min}$ (A2), which facilitates the formation of yellow color. Initially, the absorbance values of all the samples were measured and recorded before the reaction started. The absorbance of the decolorized DPPH solution was measured at $517 \mathrm{~nm}$. The DPPH reducing activity of the AgNPs was calculated based on the difference between $\mathrm{A} 1$ and $\mathrm{A} 2$ absorbance values. For control, the antioxidant activity of ascorbic acid was determined by the DDPH assay at the concentration of $160 \mu \mathrm{g} / \mathrm{ml}$, and the value was assumed as $100 \%$. The experiment was repeated thrice, and the antioxidant activity was expressed as ascorbic acid equivalent.

\section{Reducing Power Measurement Assay}

Briefly, $0.1 \mathrm{ml}$ of $1 \%(\mathrm{w} / \mathrm{v})$ potassium ferricyanide and $0.1 \mathrm{ml}$ of the synthesized AgNPs at various concentrations $(10,20,40,80$, and $160 \mu \mathrm{g} / \mathrm{ml})$ were mixed and incubated at room temperature for $30 \mathrm{~min}$. To this mixture, 10\% TCA (trichloroacetic acid) and 0.1\% (w/v) ferric chloride were added and mixed thoroughly. This reaction mixture was incubated at room temperature for $20 \mathrm{~min}$, and the absorbance was recorded at $665 \mathrm{~nm}$ in a spectrophotometer. For control, the antioxidant activity of ascorbic acid was determined at the concentration of $160 \mu \mathrm{g} / \mathrm{ml}$, and the value was assumed as $100 \%$. The experiment was performed thrice, and the antioxidant activity was expressed as ascorbic acid equivalent.

\section{Ferric reducing antioxidant power (FRAP) Assay}

The FRAP reaction mixture was prepared by adopting the protocol of Arora and Chandra, (2011). The mixture contained $300 \mathrm{mM}$ acetate buffer (pH 3.6), $10 \mathrm{mM}$ 2,4,6-tripyridyl-s-triazine (TPTZ), and $20 \mathrm{mM}$ ferric chloride in a ratio of $10: 1: 1$. For the FRAP assay, $0.5 \mathrm{ml}$ of $\operatorname{AgNPs}(10,20,40,80$, and $160 \mu \mathrm{g} / \mathrm{ml})$ were mixed with $2 \mathrm{ml}$ of FRAP reagent and $1 \mathrm{ml}$ of distilled water and incubated at room temperature for $10 \mathrm{~min}$. After incubation, the formed blue color complex $\left(\mathrm{Fe}^{2+} / \mathrm{TPTZ}(2,4,6-\right.$ tripyridyl-s-triazine)) was measured at $595 \mathrm{~nm}$. Ferrous sulfate solution ( $1 \mathrm{mg} / \mathrm{ml}$ ) was used as blank, and the antioxidant potential of the sample was determined by comparing the absorbance of the sample with that of the stock solution. For control, the antioxidant activity of ascorbic acid was determined by the FRAP assay at the concentration of $160 \mu \mathrm{g} / \mathrm{ml}$, and the value was assumed as $100 \%$. The experiment was performed thrice, and the antioxidant activity was expressed as ascorbic acid equivalent.

\section{Results and discussion}

\section{Synthesis and characterization of AgNPS}

In this study, quercetin-mediated synthesis of AgNPs was demonstrated by the rapid one-pot method. The 


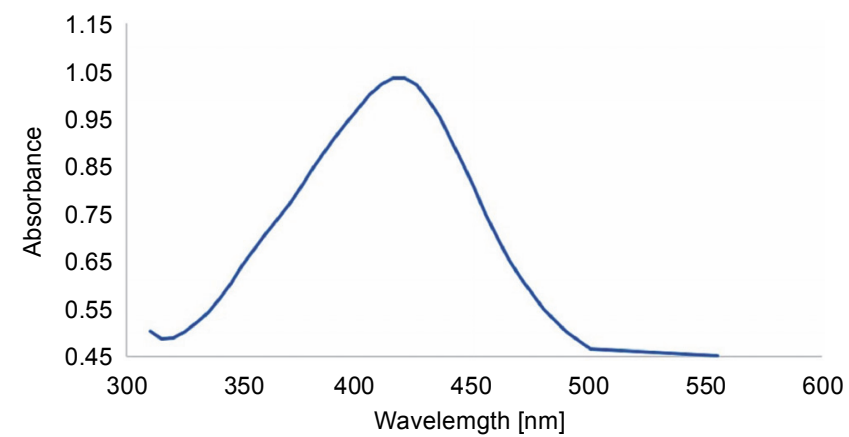

Fig. 1. Surface plasmon resonance of AgNPs observed at $420 \mathrm{~nm}$ confirms the presence of colloidal AgNPs in solution

synthesized nanoparticles were characterized by UV-VIS spectroscopy, FT-IR, SEM, and TEM. As previously shown, AgNPs exhibit antiviral (Lara et al., 2010; Morris et al., 2019), antibacterial (Behravan et al., 2019), antioxidant and antitumor (AlSalhi et al., 2019; Anandan et al., 2019), and immunomodulatory (Pandian et al., 2018) properties. Despite many available procedures to synthesize AgNPs (physical, chemical, and biological), the biological method holds great promise. It is comparatively efficient and nontoxic, thus making it better than other methods (Seralathan et al., 2014; Chowdhury et al., 2017; Tripathi et al., 2019). It is interesting to note that AgNPs synthesized using plant extracts that contain bioactive compounds such as flavonoids, alkaloids, saponins, steroids, and tannins exhibit superior pharmacological activity (Kuppusamy et al., 2016). In the present study, AgNPs were synthesized using quercetin, (a plant-derived flavonoid) (Baková and Kolesárová, 2020) by the rapid one-pot method. A promising color change (from transparent to dark brown) indicated the formation of AgNPs, specifically colloidal AgNPs. UV-VIS spectrum is mostly adopted to confirm the synthesis and stability of NPs in aqueous solutions. Figure 1 shows the absorption spectrum of AgNPs and their unique surface plasmon resonance (SPR) intense peak at $420 \mathrm{~nm}$, which was similar to that reported in our previous study (Pandian et al., 2018). A recent study (Lakshmi and Kim, 2019) reported quercetin-mediated synthesis of dual-functional gold nanoclusters for application in anticancer treatment and bioimaging. The size of the gold nanoclusters was reported as $1-3 \mathrm{~nm}$ with homogenous distribution (Lakshmi and Kim, 2019). Quercetin is a plant bioflavonoid (Baková and Kolesárová, 2020) and is a potent reducing agent (Lakshmi

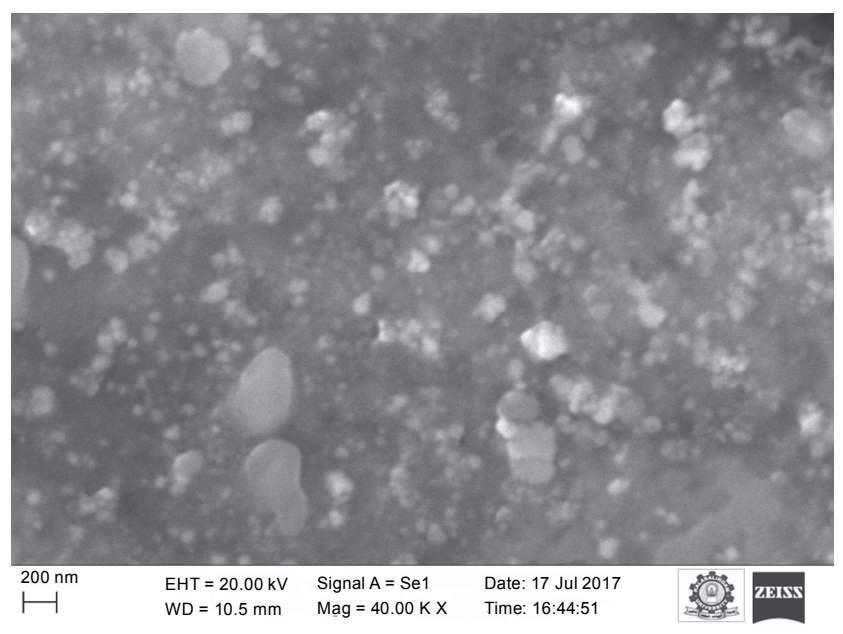

Fig. 2. Surface morphology of AgNPs visualized by SEM revealed a spherical shape

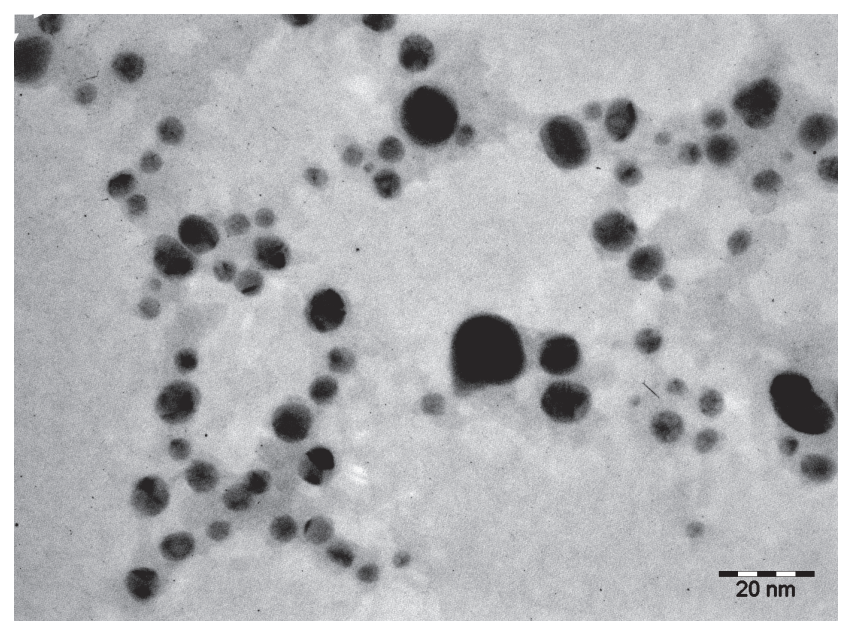

Fig. 3. TEM image of fabricated AgNPs; nonaggregated, spherical-shaped AgNPs of size 20-30 nm were observed

and Kim, 2019) with antioxidant activity. It has also been proven as a potential phytocompound for the synthesis of therapeutic metal nanoparticles (Lakshmi and Kim, 2019). Vanaraj et al. (2017) reported the average size of quercetin-based AgNPs as $65 \mathrm{~nm}$ in diameter. Interestingly, the AgNPs synthesized in our study exhibited a size of 20 to $30 \mathrm{~nm}$ (Fig. 2) with uniformly spherical shape. TEM results revealed that the AgNPs were distinct with no aggregation (Fig. 3). Ahmed and Ikram (2015) reported one-step synthesis of AgNPs with Terminalia arjuna extract and measured the average size of the synthesized AgNPs as $50 \mathrm{~nm}$. Atrocarpus altilis leaf extract was used to synthesize spherical-shaped AgNPs, and the average particle size reported was $34 \mathrm{~nm}$ (Ravi- 


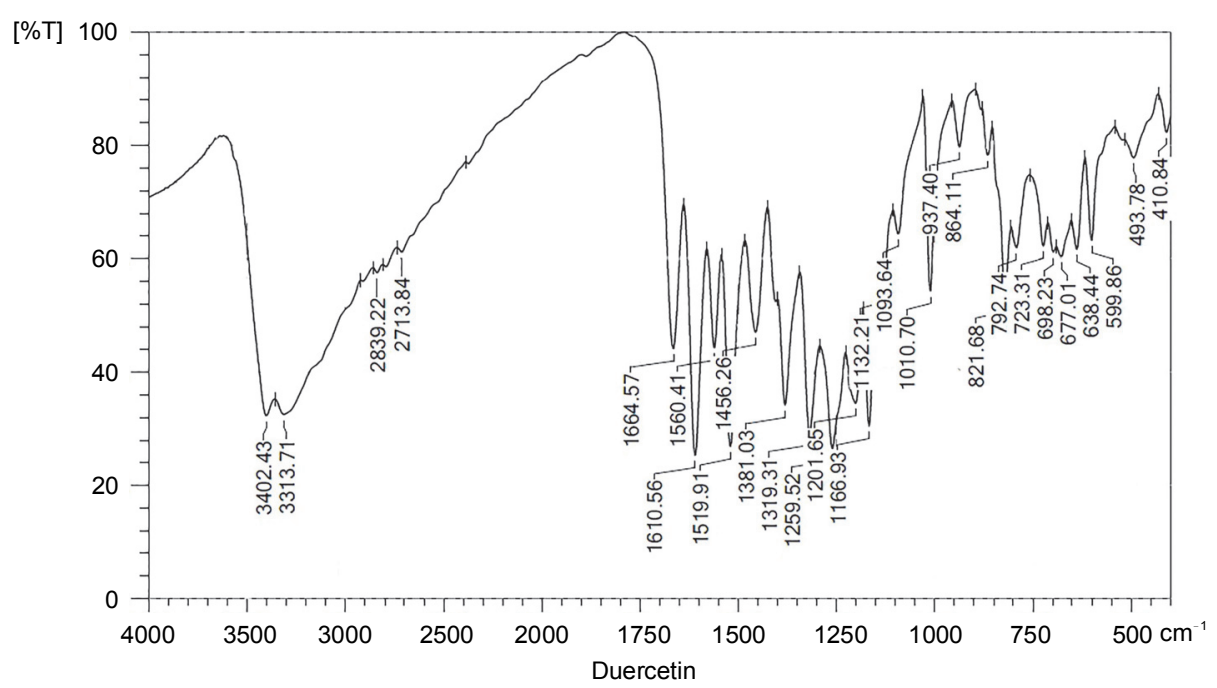

Fig. 4. Functional groups of quercetin as observed in FT-IR

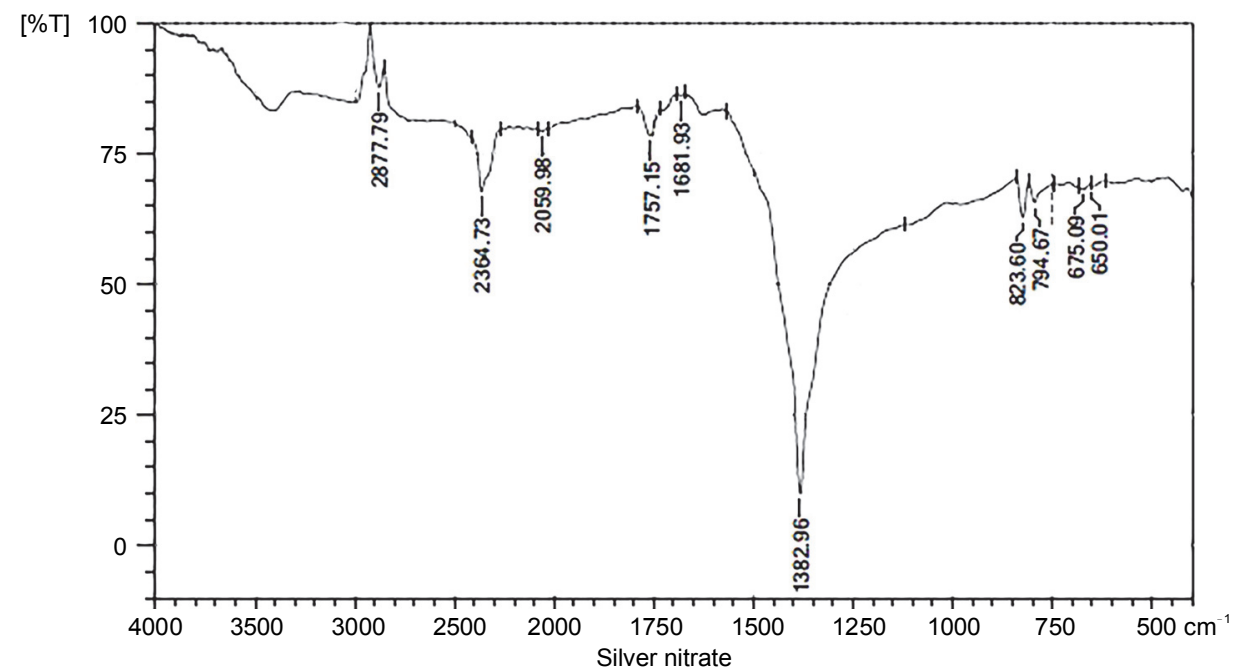

Fig. 5. Functional groups of silver nitrate as revealed in FT-IR

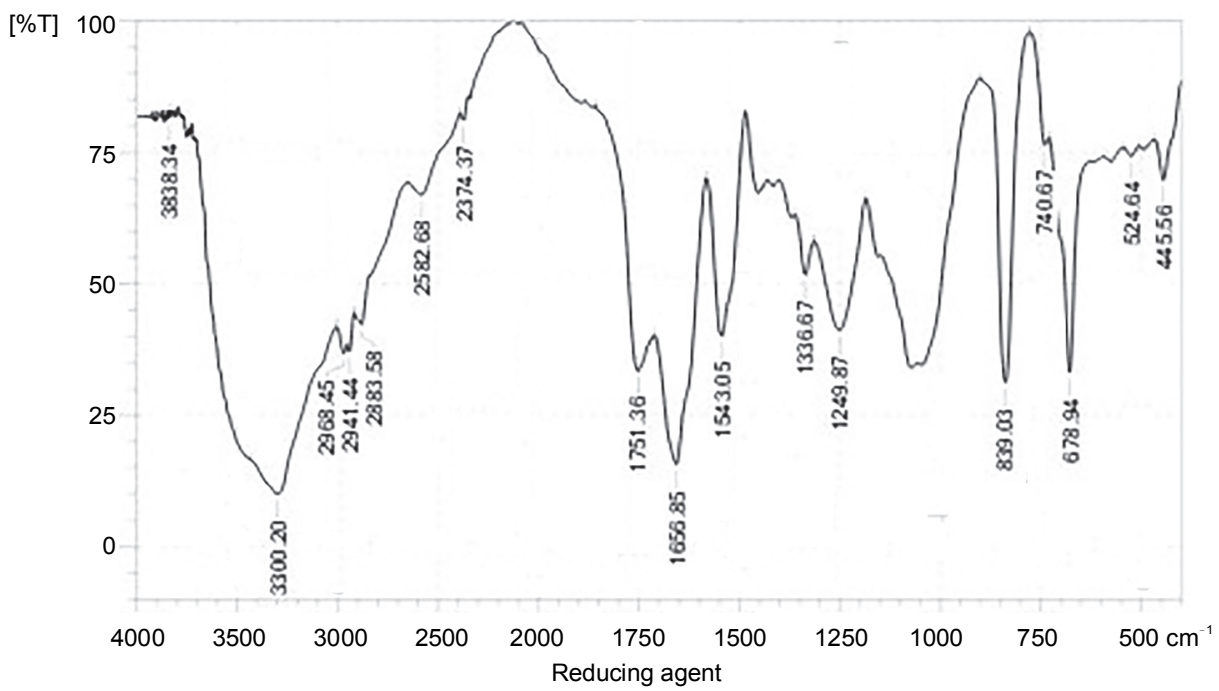

Fig. 6. FT-IR spectra of silver nanoparticles; the functional groups of silver nanoparticles and quercetin (reducing agent) are presented 
Table 1. The functional groups of quercetin as revealed in FT-IR

\begin{tabular}{|c|c|c|}
\hline \multicolumn{3}{|r|}{ Quercetin } \\
\hline number & peak & functional Group \\
\hline 1 & 638.44 & aromatic $\mathrm{C}-\mathrm{H}$ out of plane \\
\hline 2 & 677.01 & aromatic $\mathrm{C}-\mathrm{H}$ out of plane \\
\hline 3 & 698.23 & aromatic $\mathrm{C}-\mathrm{H}$ out of plane \\
\hline 4 & 723.31 & cis $\mathrm{C}-\mathrm{H}$ out of plane \\
\hline 5 & 792.74 & cis $\mathrm{C}-\mathrm{H}$ out of plane \\
\hline 6 & 821.68 & vinylidene $\mathrm{C}$-H out of plane \\
\hline 7 & 864.11 & vinylidene $\mathrm{C}-\mathrm{H}$ out of plane \\
\hline 8 & 937.40 & vinylidene $\mathrm{C}$-H out of plane \\
\hline 9 & 1010.70 & methylene $\mathrm{C}-\mathrm{H}$ bend \\
\hline 10 & 1093.64 & methylene $\mathrm{C}-\mathrm{H}$ bend \\
\hline 11 & 1132.21 & methylene $\mathrm{C}-\mathrm{H}$ bend \\
\hline 12 & 1166.93 & methylene $\mathrm{C}-\mathrm{H}$ bend \\
\hline 13 & 1201.65 & vinylidene $\mathrm{C}-\mathrm{H}$ plane bend \\
\hline 14 & 1259.52 & vinylidene $\mathrm{C}$-H plane bend \\
\hline 15 & 1319.31 & vinyl $\mathrm{C}-\mathrm{H}$ plane bend \\
\hline 16 & 1381.03 & vinyl C-H plane bend \\
\hline 17 & 1456.26 & alkyne $\mathrm{C}-\mathrm{C}$ aromatic ring stretch \\
\hline 18 & 1519.21 & alkyne $\mathrm{C}-\mathrm{C}$ aromatic ring stretch \\
\hline 19 & 1560.41 & alkyne $\mathrm{C}-\mathrm{C}$ aromatic ring stretch \\
\hline 20 & 1610.56 & alkyne $\mathrm{C}=\mathrm{C}$ aromatic ring stretch \\
\hline 21 & 1664.57 & alkyne $\mathrm{C}=\mathrm{C}$ aromatic ring stretch \\
\hline 22 & 2713.84 & methyl C-H asym./sym. stretch \\
\hline 23 & 2839.22 & methyl C-H asym./sym. stretch \\
\hline 24 & 3313.71 & $\mathrm{O}-\mathrm{H}$ broad band \\
\hline 25 & 3402.43 & $\mathrm{O}-\mathrm{H}$ broad band \\
\hline
\end{tabular}

Table 2. The functional groups of silver nitrate as observed in FT-IR

\begin{tabular}{c|c|l}
\hline \multicolumn{2}{|c}{ Silver nitrate } \\
\hline number & peak & \multicolumn{1}{c}{ functional group } \\
\hline 1 & 650.01 & aromatic C-H out of plane \\
\hline 2 & 675.09 & aromatic C-H out of plane \\
\hline 3 & 794.67 & cis C-H out of plane \\
\hline 4 & 823.60 & vinylidene C-H out of plane \\
\hline 5 & 1382.96 & alkyne C-C aromatic ring stretch \\
\hline 6 & 1681.93 & alkyne C=C aromatic ring stretch \\
\hline 7 & 1757.15 & alkyne C=C aromatic ring stretch \\
\hline 8 & 2059.98 & aromatic ring stretch \\
\hline 9 & 2364.73 & alkyne C=C bend \\
\hline 10 & 2877.79 & methyl C-H asym./sym. stretch \\
\hline
\end{tabular}

Table 3. The list of functional groups found in quercetin-functionalized AgNPs

\begin{tabular}{c|c|l}
\hline \multicolumn{3}{c}{ Silver nanoparticles } \\
\hline number & peak & \multicolumn{1}{c}{ functional group } \\
\hline 1 & 678.94 & aromatic C-H out of plane \\
\hline 2 & 740.67 & cis C-H out of plane \\
\hline 3 & 839.03 & vinylidene C-H out of plane \\
\hline 4 & 1249.87 & vinylidene C-H plane bend \\
\hline 5 & 1336.67 & vinyl C-H plane bend \\
\hline 6 & 1543.05 & alkyne C-C aromatic ring stretch \\
\hline 7 & 1656.85 & alkyne C=C aromatic ring stretch \\
\hline 8 & 1751.36 & alkyne C=C stretch \\
\hline 9 & 2374.36 & alkyne C=C bend \\
\hline 10 & 2582.68 & methyl C-H stretch \\
\hline 11 & 2883.58 & methyl C-H asym./sym. stretch \\
\hline 12 & 2941.44 & methyl C-H asym./sym. stretch \\
\hline 13 & 2968.45 & methyl C-H asym./sym. stretch \\
\hline 14 & 3300.20 & O-H broad band \\
\hline 15 & 3838.34 & O-H narrow band \\
\hline & &
\end{tabular}

chandran et al., 2016). Indeed, we have previously shown (Pandian et al., 2018) that AgNPs and PEGylated AgNPs synthesized using leaf extract of Sesbania aegyptiaca were between 16 and $23 \mathrm{~nm}$ and between 25 and $28 \mathrm{~nm}$ in size, respectively. The synthesized AgNPs were also proved to show immunomodulatory and anticancer activity in vitro (Pandian et al., 2018).

\section{Functional group analysis with FT-IR}

The wave numbers of FT-IR represent various functional groups of both AgNPs and quercetin. FT-IR was applied to analyze the functional groups of reduced metal nanoparticles and biomolecules. In a previous study, FT-IR revealed the functional groups and interactions of metal nanoparticles and biomolecules (Kunjiappan et al., 2014). The FT-IR spectra of quercetin, silver nitrate, and AgNPs are presented in Figures 4, 5, and 6 , respectively. The names of the functional groups of quercetin, silver nitrate, and AgNPs are presented in Tables 1, 2, and 3, respectively. FT-IR analysis of AgNPs showed bands at $3439,2360,2365,2881,1649,1471$, 1251,1103 , and $950 \mathrm{~cm}^{-1}$. The peaks at 1471 and $1251 \mathrm{~cm}^{-1}$ were reported to be aldehydic-C-H stretching and alcoholic-C-O stretching, respectively (Balogun et al., 2015). It was also observed that the conversion of 
A

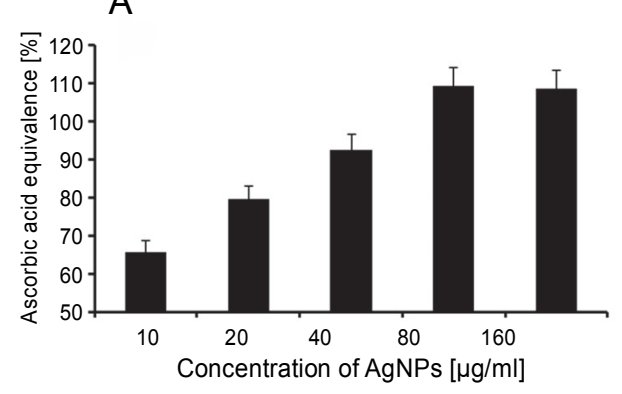

B

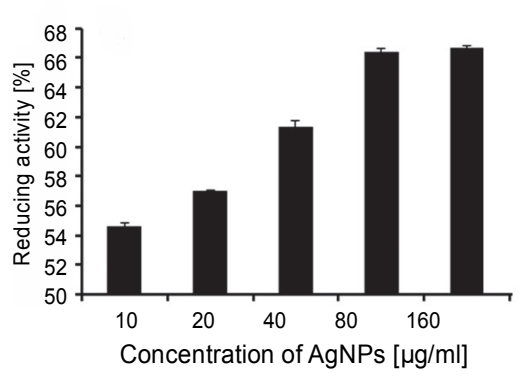

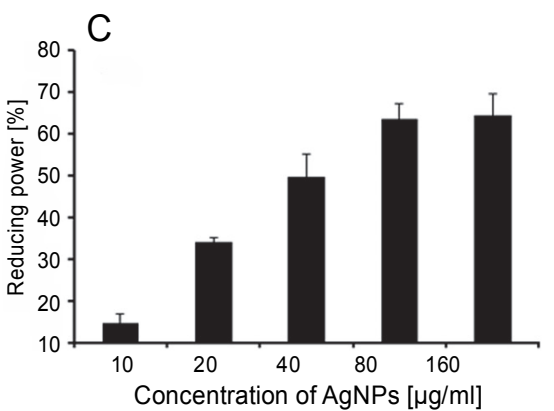

Fig. 7. In vitro antioxidant activity of AgNPs determined by A) DPPH, B) FRAP, and C) power reducing assays

broad ligands $(-\mathrm{OH})$ to soft ligands $(-\mathrm{C}-\mathrm{O})$ after the reduction process created a feasible environment for soft metal $(\mathrm{Ag})$ to form co-ordination with soft carbonyl ligands (Chowdhury et al., 2014). Therefore, we attribute the reduction potential to quercetin, which was responsible for the reduction of silver nitrate to form AgNPs. This finding is in agreement with a recent study, which reported quercetin as a potential reducing agent for the synthesis of nanomaterials (Lakshmi and Kim, 2019).

\section{Antioxidant activity of quercetin-mediated synthesized AgNPS}

AgNPs synthesized using various bioflavonoids exert antioxidant activities (Chowdhury et al., 2014; Salari et al., 2019). In the present study, the antioxidant activity of quercetin-functionalized AgNPs was studied in vitro by DPPH, FRAP, and reducing power measurement assays (Fig. 7A-C). The free radical DPPH is decolorized in the presence of antioxidants (Hasanuzzaman et al., 2013), which hold an unpaired electron that exhibits absorbance at 515 to $517 \mathrm{~nm}$ (Ahmad et al., 2020). We found a gradual increase in the antioxidant activity of AgNPs up to $80 \mu \mathrm{g} / \mathrm{ml}$; however, the activity remains unchanged at $160 \mu \mathrm{g} / \mathrm{ml}$. The antioxidant activity of AgNPs at $80 \mu \mathrm{g} / \mathrm{ml}$ was observed as equivalent to 110,66 , and $69 \%$, by DPPH, FRAP, and reducing power measurement assays, respectively. The antioxidant activity of ascorbic acid (control) was determined at the concentration of $160 \mu \mathrm{g} / \mathrm{ml}$, and the value was assumed as $100 \%$. The results of antioxidant activities were projected with ascorbic acid equivalence as demonstrated by previous scientific studies (Ahmad et al., 2020; Soltanabad et al., 2020). These results demonstrate a dose-dependent antioxidant activity of quercetin-functionalized NPs. The assays that we adopted to measure the anti- oxidant status have been widely used by other researchers (Ahmad et al., 2020; Soltanabad et al., 2020). The same assays were used to prove the antioxidant activities of apple fruit extracts and 2-phenoxypyridotriazolo pyrimidines in vitro (Abuelizz et al., 2020; Sethi et al., 2020).

Several studies have confirmed the antioxidant and anticancer potential of AgNPs under in vitro and in vivo conditions (Sre et al., 2015; Salehi et al., 2016; Khorrami et al., 2018; Mousavi et al., 2018). In the present study, we also demonstrated that the synthesized AgNPs act as potential antioxidant molecules, specifically at the concentration of $80 \mu \mathrm{g} / \mathrm{ml}$. The antioxidant, antibacterial, and cytotoxic effects of Piper longum fruit-based AgNPs were also proved in vitro (Reddy et al., 2014). It was further revealed that the antioxidant capacity of AgNPs determined by DPPH and reducing power assays differed due to various concentrations of AgNPs (100 to $600 \mu \mathrm{g} / \mathrm{ml}$ ) (Reddy et al., 2014). Ravichandran et al. (2016) reported the antioxidant activity of AgNPs synthesized using Atrocarpus altilis at the concentration of $51.17 \mu \mathrm{g} / \mathrm{ml}$ (Ravichandran et al., 2016). Similarly, Cassia tora leaf extract-mediated single-step green synthesis of AgNPs, and their antioxidant activity was reported by Saravanakumar et al. (2015). The antioxidant role of AgNPs was studied in a concentration-dependent manner $(12.5-200 \mu \mathrm{g} / \mathrm{ml})$ by the DPPH scavenging assay with vitamin $\mathrm{C}$ as the standard (Saravanakumar et al., 2015). Taken together, all these results proved that AgNPs synthesized using plant sources have potential antioxidant properties, which are perhaps enhanced by the synergistic activity of the phytocomponents present in plant extracts. We therefore suggest that quercetin used in the present study could have influenced the reduction potential of silver and aided in the formation 
of AgNPs, which eventually culminates in the exhibition of antioxidant property.

\section{Conclusions}

In the present study, quercetin was used to synthesize AgNPs by the one-pot method. The synthesized AgNPs were $20-30 \mathrm{~nm}$ in size and spherical in shape. The significance of this work lies in the fabrication of AgNPs by using a single-step method and its extended stability at room temperature. The synthesized AgNPs were demonstrated to exert potential antioxidant properties in vitro. We conclude that phytochemical-mediated synthesis of metal NPs would be advantageous because it provides extended stability and synergistically improves their pharmacological properties.

\section{Acknowledgments}

The authors are grateful to Dr. Devaraj Sankarganesh, Postdoctoral Researcher, Department of Microbial Biotechnology, Bharathiar University, Coimbatore, India, for critically evaluating the manuscript and providing suggestions. We thank the technicians, Mr. P. Kathirvel and Mr. V. Krishnaprabhu, Sir C.V. Raman K.S. Krishnan International Research Center, Kalasalingam Academy of Research and Education, Krishnankoil, India, for their assistance in characterization studies (SEM, FT-IR, and UV-VIS spectrophotometry). We also thank Ms. Ewa Babkiewicz, and Dr. Piotr Maszczyk, Department of Hydrobiology, Faculty of Biology, University of Warsaw, Warszawa, Poland, for timely help and support.

\section{References}

Abuelizz H.A., Anouar E., Marzouk M., Taie H.A.A., Ahudhaif A., Al-Salahi R. (2020) DFT study and radical scavenging activity of 2-phenoxypyridotriazolo pyrimidines by DPPH, ABTS, FRAP and reducing power capacity. Chem. Papers 74: 2893-2899.

Ahmad M., Mohammad N., Aziz M.A., Alam M.A., Hossain M.S., Islam M.R. et al. (2020) Comparison of antioxidant role of methanol, acetone and water extracts of Andrographis paniculata Nees. J. Med. Plants. Res. 14: 428-437.

Ahmed S., Ahmad M., Swami B.L., Ikram S. (2016) A review on plants extract mediated synthesis of silver nanoparticles for antimicrobial applications: a green expertise. J. Adv. Res. 7: 17-28.

Ahmed S., Ikram S. (2015) Silver nanoparticles: one pot green synthesis using Terminalia arjuna extract for biological application. J. Nanomed. Nanotechnol. 6: 309.

AlSalhi M.S., Elangovan K., Ranjitsingh A.J.A., Murali P., Devanesan S. (2019) Synthesis of silver nanoparticles using plant derived $4-N$-methyl benzoic acid and evaluation of antimicrobial, antioxidant and antitumor activity. Saudi. J. Biol. Sci. 26: 970-978.

Anandan M., Poorani G., Boomi P., Varunkumar K., Anand K., Chuturgoon A.A. et al. (2019) Green synthesis of aniso- tropic silver nanoparticles from the aqueous leaf extract of Dodonaea viscosa with their antibacterial and anticancer activities. Process. Biochem. 80: 80-88.

Arora D.S., Chandra P. (2011) In vitro antioxidant potential of some soil fungi: screening of functional compounds and their purification from Penicillium citrinum. Appl. Biochem. Biotechnol. 165: 639.

Azharuddin M., Zhu G.H., Das D., Ozgur E., Uzun L., Turner A.P. et al. (2019) A repertoire of biomedical applications of noble metal nanoparticles. Chem. Commun. 55: 6964-6996.

Baková Z., Kolesárová A. (2020) Bioflavonoid quercetin-food sources, bioavailability, absorbtion and effect on animal cells. J. Microbiol. Biotechnol. Food. Sci. 9: 426-433.

Balachandar R., Gurumoorthy P., Karmegam N., Barabadi H., Subbaiya R., Anand K. et al. (2019) Plant-mediated synthesis, characterization and bactericidal potential of emerging silver nanoparticles using stem extract of Phyllanthus pinnatus: a recent advance in phytonanotechnology. J. Clust. Sci. 30: 1481-1488.

Balogun A.O., Lasode O.A., Li H., McDonald A.G. (2015) Fourier transform infrared (FTIR) study and thermal decomposition kinetics of Sorghum bicolour glume and Albizia pedicellaris residues. Waste Biomass Valor. 6: 109-116.

Banu H., Renuka N., Faheem S.M., Ismail R., Singh V., Saadatmand Z. et al. (2018) Gold and silver nanoparticles biomimetically synthesized using date palm pollen extractinduce apoptosis and regulate $p 53$ and Bcl-2 expression in human breast adenocarcinoma cells. Biol. Trace. Elem. Res. 186: 122-134.

Bawaskar M., Gaikwad S., Ingle A., Rathod D., Gade A., Duran N. et al. (2010) A new report on mycosynthesis of silver nanoparticles by Fusarium culmorum. Curr. Nanosci. 6: 376-380.

Behravan M., Panahi A.H., Naghizadeh A., Ziaee M., Mahdavi R., Mirzapour A. (2019) Facile green synthesis of silver nanoparticles using Berberis vulgaris leaf and root aqueous extract and its antibacterial activity. Int. J. Biol. Macromol. 124: 148-154.

Borase H.P., Salunke B.K., Salunkhe R.B., Patil C.D., Hallsworth J.E., Kim B.S. et al. (2014) Plant extract: a promising biomatrix for ecofriendly, controlled synthesis of silver nanoparticles. Appl. Biochem. Biotechnol. 173: 1-29.

Chen D., Qiao X., Qiu X., Chen J. (2009) Synthesis and electrical properties of uniform silver nanoparticles for electronic applications. J. Mater. Sci. 44: 1076-1081.

Chowdhury A., Kunjiappan S., Bhattacharjee C., Chowdhury R. (2014) Green synthesis and characterization of biocompatible silver nanoparticles using Brassica oleracea $L$. leaf extract. Int. J. Curr. Res. 6: 6166-6174.

Chowdhury A., Kunjiappan S., Bhattacharjee C., Somasundaram B., Panneerselvam T. (2017) Biogenic synthesis of Marsilea quadrifolia gold nanoparticles: a study of improved glucose utilization efficiency on 3T3-L1 adipocytes. Vitr. Cell. Dev. Biol. 53: 483-493.

Dajas F. (2012) Life or death: neuroprotective and anticancer effects of quercetin. J. Ethnopharmacol. 143: 383-396. 
Evanoff Jr, D.D., Chumanov G. (2005) Synthesis and optical properties of silver nanoparticles and arrays. Chem. Phys. Chem. 6: 1221-1231.

Hasanuzzaman M., Ali M.R., Hossain M., Kuri S., Islam M.S. (2013) Evaluation of total phenolic content, free radical scavenging activity and phytochemical screening of different extracts of Averrhoa bilimbi (fruits). Int. Curr. Pharm J. 2: 92-96.

Ingale A.G., Chaudhari A.N. (2013) Biogenic synthesis of nanoparticles and potential applications: an eco-friendly approach. J. Nanomed. Nanotechol. 4: 1-7.

Jemilugba O.T., Parani S., Mavumengwana V., Oluwafemi O.S. (2019) Green synthesis of silver nanoparticles using Combretum erythrophyllum leaves and its antibacterial activities. Colloid. Interface Sci. Commun. 31: 100191.

Khorrami S., Zarrabi A., Khaleghi M., Danaei M., Mozafari M.R. (2018) Selective cytotoxicity of green synthesized silver nanoparticles against the MCF-7 tumor cell line and their enhanced antioxidant and antimicrobial properties. Int. J. Nanomed. 13: 8013.

Krishnaraj C., Ramachandran R., Mohan K., Kalaichelvan P.T. (2012) Optimization for rapid synthesis of silver nanoparticles and its effect on phytopathogenic fungi. Spectrochim. Acta A: Mol. Biomol. Spectrosc. 93: 95-99.

Kunjiappan S., Chowdhury R., Bhattacharjee C. (2014) A green chemistry approach for the synthesis and characterization of bioactive gold nanoparticles using Azolla microphylla methanol extract. Front. Mater. Sci. 8: 123-135.

Kuppusamy P., Yusoff M.M., Maniam G.P., Govindan N. (2016) Biosynthesis of metallic nanoparticles using plant derivatives and their new avenues in pharmacological applications - an updated report. Saudi. Pharm. J. 24: 473-484.

Lakshmi B.A., Kim S. (2019) Quercetin mediated gold nanoclusters explored as a dual functional nanomaterial in anticancer and bio-imaging disciplines. Colloids Surf. B: Biointerfaces 178: 230-237.

Lara H.H., Ayala-Nuñez N.V, Ixtepan-Turrent L., RodriguezPadilla C. (2010) Mode of antiviral action of silver nanoparticles against HIV-1. J. Nanobiotech. 8: 1.

Lesjak M., Beara I., Simin N., Pintać D., Majkić T., Bekvalac K. et al. (2018) Antioxidant and anti-inflammatory activities of quercetin and its derivatives. J. Funct. Foods. 40: 68-75.

Lin J., He C., Zhao Y., Zhang S. (2009) One-step synthesis of silver nanoparticles/carbon nanotubes/chitosan film and its application in glucose biosensor. Sensors Actuators B: Chem. 137: 768-773.

Mahmoudi R., Aghaei S., Salehpour Z., Mousavizadeh A., Khoramrooz S.S., Taheripour Sisakht M. et al. (2020) Antibacterial and antioxidant properties of phyto-synthesized silver nanoparticles using Lavandula stoechas extract. Appl. Organomet. Chem. 34: e5394.

Masum M., Islam M., Siddiqa M., Ali K.A., Zhang Y., Abdallah Y. et al. (2019) Biogenic synthesis of silver nanoparticles using Phyllanthus emblica fruit extract and its inhibitory action against the pathogen Acidovorax oryzae Strain RS-2 of rice bacterial brown stripe. Front. Microbiol. 10: 820.
Mittal A.K., Chisti Y., Banerjee U.C. (2013) Synthesis of metallic nanoparticles using plant extracts. Biotech. Adv. 31: 346-356.

Morris D., Ansar M., Speshock J., Ivanciuc T., Qu Y., Casola A. et al. (2019) Antiviral and immunomodulatory activity of silver nanoparticles in experimental RSV infection. Viruses 11: 732.

Mousavi S.M., Hashemi S.A., Ghasemi Y., Atapour A., Amani A.M., Savar Dashtaki A. et al. (2018) Green synthesis of silver nanoparticles toward bio and medical applications: review study. Artif. Cells Nanomed. Biotech. 46: S855-S872.

Nikaeen G., Yousefinejad S., Rahmdel S., Samari F., Mahdavinia S. (2020) Central composite design for optimizing the biosynthesis of silver nanoparticles using Plantago major extract and investigating antibacterial, antifungal and antioxidant activity. Sci. Rep. 10: 1-16.

Pandian S.R.K., Anjanei D., Raja N.R.L., Sundar K. (2018) PEGylated silver nanoparticles from Sesbania aegyptiaca exhibit immunomodulatory and anti-cancer activity. Mater. Res. Express. 6: 35402.

Pandian S.R.K., Deepak V., Kalishwaralal K., Viswanathan P., Gurunathan S. (2010) Mechanism of bactericidal activity of silver nitrate - a concentration dependent bi-functional molecule. Brazil. J. Microbiol. 41: 805-809.

Rajan R., Chandran K., Harper S.L., Yun S.-I., Kalaichelvan P.T. (2015) Plant extract synthesized silver nanoparticles: an ongoing source of novel biocompatible materials. Ind. Crops. Prod. 70: 356-373.

Ravichandran V., Vasanthi S., Shalini S., Shah S.A.A., Harish R. (2016) Green synthesis of silver nanoparticles using Atrocarpus altilis leaf extract and the study of their antimicrobial and antioxidant activity. Mater. Lett. 180: 264-267.

Reddy N.J., Vali D.N., Rani M., Rani S.S. (2014) Evaluation of antioxidant, antibacterial and cytotoxic effects of green synthesized silver nanoparticles by Piper longum fruit. Mater. Sci. Eng. C. 34: 115-122.

Ren Y., Yang H., Wang T., Wang C. (2019) Bio-synthesis of silver nanoparticles with antibacterial activity. Mater. Chem. Phys. 235: 121746.

Salari S., Esmaeilzadeh Bahabadi S., Samzadeh-Kermani A., Yosefzaei F. (2019) In-vitro evaluation of antioxidant and antibacterial potential of green synthesized silver nanoparticles using Prosopis farcta fruit extract. Iran. J. Pharm. Res. 18: 430-455.

Salehi S., Shandiz S.A.S., Ghanbar F., Darvish M.R., Ardestani M.S., Mirzaie A. et al. (2016) Phytosynthesis of silver nanoparticles using Artemisia marschalliana Sprengel aerial part extract and assessment of their antioxidant, anticancer, and antibacterial properties. Int. J. Nanomed. 11: 1835.

Saravanakumar A., Ganesh M., Jayaprakash J., Jang H.T. (2015) Biosynthesis of silver nanoparticles using Cassia tora leaf extract and its antioxidant and antibacterial activities. J. Ind. Eng. Chem. 28: 277-281.

Saxena J., Sharma P.K., Sharma M.M., Singh A. (2016) Process optimization for green synthesis of silver nanoparti 
cles by Sclerotinia sclerotiorum MTCC 8785 and evalua tion of its antibacterial properties. Springerplus 5: 1-10.

Seralathan J., Stevenson P., Subramaniam S., Raghavan R., Pemaiah B., Sivasubramanian A. et al. (2014) Spectroscopy investigation on chemo-catalytic, free radical scavenging and bactericidal properties of biogenic silver nanoparticles synthesized using Salicornia brachiata aqueous extract. Spectrochim. Acta A: Mol. Biomol. Spectrosc. 118: 349-355.

Sethi S., Joshi A., Arora B., Bhowmik A., Sharma R.R., Kumar P. (2020) Significance of FRAP, DPPH, and CUPRAC assays for antioxidant activity determination in apple fruit extracts. Eur. Food. Res. Technol. 246: 591-598.

Singh S.P., Bhargava C.S., Dubey V., Mishra A., Singh Y. (2017) Silver nanoparticles: biomedical applications, toxicity, and safety issues. Int. J. Res. Pharm. Pharm. Sci. 4: 1-10.

Soltanabad M.H., Bagherieh-Najjar M.B., Mianabadi M. (2020) Carnosic acid content increased by silver nanoparticle Treatment in rosemary (Rosmarinus officinalis L.). Appl. Biochem. Biotech. 191: 482-495.

Sre P.R.R., Reka M., Poovazhagi R., Kumar M.A., Murugesan K. (2015) Antibacterial and cytotoxic effect of biologically synthesized silver nanoparticles using aqueous root extract of Erythrina indica lam. Spectrochim. Acta. A: Mol. Biomol. Spectrosc. 135: 1137-1144.
Taha Z.K., Hawar S.N., Sulaiman G.M. (2019) Extracellular biosynthesis of silver nanoparticles from Penicillium italicum and its antioxidant, antimicrobial and cytotoxicity activities. Biotech. Lett. 41: 899-914.

Tripathi D., Modi A., Narayan G., Rai S.P. (2019) Green and cost effective synthesis of silver nanoparticles from endangered medicinal plant Withania coagulans and their potential biomedical properties. Mater. Sci. Eng. C 100: 152-164.

Umaralikhan L., Jaffar M.J.M. (2018) Green synthesis of MgO nanoparticles and it antibacterial activity. Iran. J. Sci. Technol. Trans. A: Sci. 42: 477-485.

Vanaraj S., Keerthana B.B., Preethi K. (2017) Biosynthesis, characterization of silver nanoparticles using quercetin from Clitoria ternatea $L$. to enhance toxicity against bacterial biofilm. J. Inorg. Organomet. Polym. Mater. 27: 1412-1422.

Virkutyte J., Varma R.S. (2011) Green synthesis of metal nanoparticles: biodegradable polymers and enzymes in stabilization and surface functionalization. Chem. Sci. 2: 837-846.

Wei L., Lu J., Xu H., Patel A., Chen Z.-S., Chen G. (2015) Silvernanoparticles: synthesis, properties, and therapeutic applications. Drug. Discov. Today. 20: 595-601. 